\title{
Types for Hierarchic Shapes ${ }^{\star}$ (Summary)
}

\author{
Sophia Drossopoulou ${ }^{1}$, Dave Clarke ${ }^{2}$, and James Noble ${ }^{3}$ \\ ${ }^{1}$ Imperial College London, UK \\ ${ }^{2}$ CWI, Amsterdam, The Netherlands \\ ${ }^{3}$ Victoria University of Wellington, Wellington, NZ
}

\begin{abstract}
Heap entities tend to contain complex references to each other. To manage this complexity, types which express shapes and hierarchies have been suggested. We survey type systems which describe such hierarchic shapes, how these types are used for reasoning about programs, and applications in concurrent programming.
\end{abstract}

Most imperative programs create and manipulate heap entities (objects, or records) which contain references to each other forming intricate topologies. This creates complexity, and makes programs difficult to understand and manipulate. Programmers, on the other hand, tend to think in terms of shapes, categorizations and hierarchies.

Thus, in the last decade, types describing shapes and hierarchies have been proposed to express programming intuitions, to support verification, and for synchronization and optimizations. We will discuss types for hierarchic shapes in terms of object oriented programming, because, even though the ideas are applicable to any imperative language, most of the related research was conducted in the context of object oriented languages.

\section{Types for Hierarchic Shapes}

Information hiding 28] was suggested as early as the 1970s, as a means to make programs more robust and easy to understand. Mechanisms that achieve information hiding by restricting the visibility of names, e.g., private/protected annotations, are useful but insufficient. They prevent the name of an entity from being used outside a class or package, but do not prevent a reference to an entity from being leaked out of a structure [26].

To prevent such leaking of references, type systems have been suggested which give guarantees about the topology of the object graph, i.e., about which objects may access which other objects.

Ownership types [15] introduce the concept of an object owning its nested objects; an ownership context is the set of objects with a given common owner.

\footnotetext{
* Slides available from slurp.doc.ic.ac.uk/pubs.html\#esop06.
} 
Objects have a unique owner, thus ownership contexts are organized hierarchically into a tree structure. Furthermore, the owner controls access to the owned objects, because an object may only be accessed by its direct owner, or by objects (possibly indirectly) owned by the former object's owner. Therefore, owners are dominators [15, where $o_{1}$ dominates $o_{2}$, if any path from the "outside" (or "root" of the object graph) to $o_{2}$ goes through $o_{1}$.

Ownership types can thus be used to characterize the runtime structure of object graphs. Analysis of the heaps of programs has demonstrated that indeed, object graphs tend to have structure: In [29] analysis of heap dumps for a corpus of programs demonstrated that the average nesting (ownership) depth of objects is 5 . In [30] heap dumps for 60 object graphs from 35 programs demonstrated that the number of incoming and outgoing references follow a power law, whereby the $\log$ of the number of objects with $k$ references is proportional to $\log$ of $k$, thus challenging the common perception that oriented programs are built out of layers of homogeneous components.

The owners as dominators approach, also known as deep ownership, gives very strong encapsulation properties which are natural in containers and nested structures [13. The approach has been used in program visualization [25].

On the other hand, deep ownership makes coding some popular structures, notably iterators, rather cumbersome. To alleviate this, shallow ownership has given up on the notion of owners as dominators. In [10 inner classes have privileged access to the objects enclosed by the corresponding outer class object; i.e., objects of an inner class may refer to objects owned by their outer class objects. In [14,2] objects on the stack are allowed to break deep ownership, and to refer to the inside of an ownership context. A more refined approach [1] decouples the encapsulation policy from the ownership mechanism, by allowing multiple ownership domains (contexts in our terminology) per object, and by allowing the programmer to specify permitted aliasing between pairs of contexts.

Ownership types usually cannot easily handle change of owner, except for externally unique objects, i.e., for objects for which references from the outside are unique [17.

The type of an object describes the owner of the object itself as well as the owners of the fields of the object; because these may be distinct, types are parameterized by ownership parameters which will be instantiated by objects enclosing the current object. This requires all types to be annotated by a number of ownership parameters.

Universes [22] suggest a more lightweight approach, whereby references to owned objects, or references to objects with the same owner may be used for modifications, and references to any other objects are readonly. Thus, universe type systems do not require ownership parameters, and instead only distinguish between rep for owned, peer for same owner, and readonly annotations. Types are coarser: readonly are readonly references which may point into any context.

Confined types, on the other hand, introduce the concept of classes confined to their defining package, and guarantee that instances of a confined class are only accessible by instances of classes from the same package; thus, they are only 
manipulated by code belonging to the same package as the class [8]. The annotations required for confined types are simple, and the object graph structure is simpler in the sense that the ownership contexts represent the packages, and thus are statically known.

\subsection{Hierarchic Shapes for Program Verification}

The decomposition of heaps into disjoint sets of objects allows these objects to be treated together for the purposes of verification. Central issues in the context of program verification are that an object's properties may depend on other objects' properties, that objects' invariants need to be temporarily broken and later re-established, and the treatment of abstraction layers, e.g., when a Set is implemented in terms of a List. The notion of ownership is primarily related to the dependence of objects' properties rather than the topology of object graphs.

Universes were developed with the aim to support modular program verification; in 24] universe types were applied to JML for the description of frame properties, where modifies clauses of method specifications define which objects may be modified. Modularity is achieved by a form of "underspecification" of the semantics, allowing method calls to modify objects outside the ownership context of the receiver without being mentioned in the relevant modifies-clause.

In [6] a methodology for program specification and verification is proposed, whereby an object's invariants may depend on (possibly indirectly) owned objects. The state space of programs is enriched to express whether an object's validity holds (i.e., whether its invariant holds); there is support for explicitly altering an object validity, and explicit ownership transfer. Subclassing means that an object's invariant may hold at the level of different superclasses of the given object. This approach is refined and adapted to universes in 21], and is implemented in Boogie, and further extended in [7] to allow invariants to be expressed over shared state.

However, the necessity to explicitly manipulate an object's validity increases the overhead of verification; therefore, 23. defines implicitly in which execution states an object's invariants must hold, based on an ownership model which is enforced by the type system.

Representation independence, which means that a class can safely be replaced by another "equivalent" class provided it is encapsulated, i.e., its internal representation is owned by instances of that class, is proven in [4]. In [5] the approach is extended to deal with shared state, recursive methods and callbacks, and the application to program equivalence.

In a more fundamental approach, [20] develops a logic for reasoning about mutable data structures whereby the spatial conjunction operator $*$ splits the heap into two disjoint parts, usually one representing the part necessary for some execution, and the other representing the rest. In [19] the conjunction * is used to separate the internal resources of a module from those accessed by its client, to support verification in the context of information hiding. Work in 27. introduces abstract predicates, which are treated atomically outside a data structure, but whose definition may be used within the data structure, thus 
supporting reasoning about modules, ADTs and classes. In these approaches the heap is split afresh in each verification step; there is no hierarchy in that the heap is just split into two parts. The approaches are very flexible, but do not yet handle issues around the dependency of objects' properties and breaking/reestablishing of objects' invariants.

Using a simpler methodology, rather than attempt full-fledged verification, [14] describes read-write effects of methods in terms of the ownership contexts, and uses these to determine when method calls are independent, i.e., their execution does affect each other. In 31 the approach is extended to describe read-effects of predicates, and to infer when some execution does not affect the validity of some predicate.

\subsection{Applications of Hierarchic Shapes}

Hierarchic shapes have successfully been applied in concurrent programming, garbage collection, and in deployment time checks of architectural invariants.

Guava [3] introduces additional type rules to Java which control synchronization by distinguishing between objects which can be shared across threads, and those which cannot. The former are monitors, and the latter are either thread-local, or encapsulated within a monitor.

In 11] race-free programs are obtained though an extension of ownership types, where an object may be owned not only by another object (as in the classical system) but also by the object itself, or by a thread (to express objects local to threads). By acquiring the lock at the root of an ownership tree, a thread acquires exclusive access to all the members of that tree. In [9] the approach is extended to prevent deadlocks, by requiring a partial order among all locks, and statically checking that threads holding more than one lock acquire them in descending order.

In real-time Java, timely reclamation of memory is achieved through scoped types 32,12. Scopes correspond to ownership contexts, in that they contain objects, are hierarchically organized into a tree, and outer scopes may not hold references to objects within more deeply nested inner scopes. When a thread working in scope $S_{1}$ enters scope $S_{2}$, then $S_{1}$ becomes the owner of $S_{2}$. When a thread enters a scope it is dynamically checked that it originated in its owner scope, thus guaranteeing nesting of scopes into a tree hierarchy. Scopes are released upon thread exit.

In [16] the architectural integrity constraints of the Enterprise Java Beans architecture, which require beans to be confined within their wrappers, are enforced through a lightweight confinement model and a deployment checker.

\subsection{Inference of Hierarchic Shapes}

The various systems for hierarchic shapes impose an extra burden of annotation to programmers, as they require each appearance of a class in a type description to be annotated by ownership parameters or restrictions such as rep. 
Kacheck/J [18] is a tool which infers which classes are confined within a package in the sense of [8]. Applied on a corpus of 46,000 classes, it could deduce that around $25 \%$ of package scoped classes are confined.

In [2] an algorithm to infer ownership types is developed and successfully applied to 408 classes of the Java standard library. However, inferred types often contain too many ownership parameters, so precision needs to be improved.

\section{Conclusions}

Hierarchic shapes have successfully been used for program visualization and verification, in concurrent programming, garbage collection, and for architectural integrity constraints. Hierarchic shapes come in different flavours, and differ in whether they support change of owner, whether ownership implies restrictions on aliasing (through deep or shallow ownership) or dependence of properties, whether the ownership contexts correspond to objects, classes or packages, whether the number of ownership contexts is statically or dynamically known, whether ownership is checked statically or dynamically, how many annotations are required, and whether inference is supported.

Further work is required to combine the different uses of the shapes, to develop more lightweight yet powerful systems, to develop better inference tools to alleviate the process of annotating programs, to combine shape types with new trends in program development (most notably with aspect oriented programming), and finally to combine the ease of use offered by types with the flexibility offered by full-fledged verification as in separation logic.

\section{References}

1. Jonathan Aldrich and Craig Chambers. Ownership Domains: Separating aliasing Policy from Mechanism. In ECOOP, 2004.

2. Jonathan Aldrich, Valentin Kostadinov, and Craig Chambers. Alias Annotations for Program Understanding. In OOPSLA, November 2002.

3. David F. Bacon, Robert E. Strom, and Ashis Tarafdar. Guava: a dialect of Java without data races. In OOPSLA, 2000.

4. Anindya Banerjee and David A. Naumann. Ownership confinement ensures representation independence for object-oriented programs. JACM, 2005.

5. Anindya Banerjee and David A. Naumann. State based ownership, renetrnace and encapsulation. In ECOOP, 2005.

6. Mike Barnett, Robert DeLine, Manuel Fähndrich, K. Rustan M. Leino, and Wolfram Schulte. Verification of Object-Oriented Programs with Invariants. Journal of Object Technology, 2004.

7. Mike Barnett and David A. Naumann. Freinds Need a Bit More: Maintaining Invariants Over Shared State. In Mathematics of Program Construction, 2004.

8. Boris Bokowski and Jan Vitek. Confined Types. In OOPSLA, 1999.

9. Chandrasekar Boyapati, Robert Lee, and Martin Rinard. Ownership Types for Safe Programming: Preventing Data Races and Deadlocks. In OOPSLA, 2002.

10. Chandrasekar Boyapati, Barbara Liskov, and Liuba Shrira. Ownership Types for Object Encapsulation. In POPL, 2003. 
11. Chandrasekar Boyapati and Martin Rinard. A Parameterized Type System for Race-Free Java Programs. In OOPSLA, 2002.

12. Chandrasekhar Boyapati, Alexandru Salcianu, William Beebee, and Martin Rinard. Ownership Types for Safe Region-Based Memory Management in Real-Time Java. In PLDI, June 2003.

13. Gustaf Cele and Sebastian Stureborg. Ownership Types in Practice. Technical Report TR-02-02, Stockholm University, 2002.

14. David Clarke and Sophia Drossopolou. Ownership, Encapsulation and the Disjointness of Type and Effect. In OOPSLA, 2002.

15. David Clarke, John Potter, and James Noble. Ownership Types for Flexible Alias Protection. In OOPSLA, 1998.

16. David Clarke, Michael Richmond, and James Noble. Saving the world from bad beans: Deployment-time confinement checking. In OOPSLA, 2003.

17. David Clarke and Tobias Wrigstad. External Uniqueness is Unique Enough. In ECOOP, 2003.

18. Christian Grothoff, Jens Palsberg, and Jan Vitek. Encapsulating Objects with Confined Types. In OOPSLA, 2001.

19. Peter W. O' Hearn, Hongseok Yang, and John C. Reynolds. Separation and information hiding. In POPL, 2004.

20. Samin Ishtiaq and Peter W. O' Hearn. Bi as an assertion language for mutable data structures. In $P O P L, 2000$.

21. K. Rustan M. Leino and Peter Müller. Object Invariants in Dynamic Contexts. In ECOOP, 2004.

22. Peter Müller and Arnd Poetzsch-Heffter. Universes: A Type System for Controlling Representation Exposure. In Programming Languages and Fundamentals of Programming, 1999.

23. Peter Müller, Arnd Poetzsch-Heffter, and Gary Leavens. Modular Invariants for Layered Object Structures. Technical Report 424, ETH Zürich, 2004. further development under way.

24. Peter Müller, Arnd Poetzsch-Heffter, and Gary T. Leavens. Modular Specification of Frame Properties in JML. Concurrency and Computation Practice and Experience, 2003.

25. James Noble. Visualising Objects: Abstraction, Encapsulation, Aliasing and Ownership. In Haim Kilov, Bernhard Rumpe, and Ian Simmonds, editors, Software Visualisation, State of the Art Survey, pages 58-72. LNCS 2269, 2002.

26. James Noble, Jan Vitek, and John Potter. Flexible Alias Protection. In ECOOP, 1998.

27. Matthew Parkinson and Gavin Bierman. Separation logic and abstraction. In POPL, 2004.

28. David Parnas. On the Criteria to be Used in Decomposing Systems into Modules. Comm. ACS, 1972.

29. Alex Potanin and James Noble. Checking ownership and confinement properties. In Formal Techniques for Java-like Programs, 2002.

30. Alex Potanin, James Noble, Marcus Frean, and Robert Biddle. Scale-free geometry in OO programs. Commun. ACM, 2005.

31. Matthew Smith and Sophia Drossopoulou. Cheaper Reasoning with Ownership Types. In IWACO. 2003.

32. Tian Zhao, James Noble, and Jan Vitek. Scoped Types for Real-time Java. In RTSS, 2004. 